\title{
The Diagnostic Value of 2D- Speckle Tracking Echocardiography for Identifying Subclinical Ventricular Dysfunction in Subjects with Early Repolarization Pattern
}

\author{
Tuğçe Çöllüoğlu ${ }^{1}$, Orhan Onalan ${ }^{1}$, and Fahri Çakan ${ }^{1}$ \\ ${ }^{1}$ Karabük University Educational and Research Hospital
}

February 17, 2021

\begin{abstract}
Introduction: Early repolarization pattern (ERP) has been known a benign electrocardiographic variant for decades. However, it can exist a silent substrate for arrhytmic events in accordance with the previous studies which have shown that there has been evidence of morphological changes in left ventricle (LV) in subjects with ERP. Despite structural changes in ERP subjects, it has not exactly known that whether a change in functional parameters of LV occur in these population. The aim of our study was to investigate LV functional parameters in subjects with ERP by the use of 2D- speckle tracking echocardiography (2D-STE). Method: In this study, subjects with ERP $(\mathrm{n}=50)$ and subjects without ERP $(\mathrm{n}=50)$ were recruited between 01.04.2018 and 01.09.2018. For each case, 2D- STE evaluation was performed by the same cardiologist. Results: Mean LV global longitudinal strain (GLS) and GLS in all apical chamber views, longitudinal peak systolic strain rate (SRS) at A3C, early diastolic strain rate (SRE) at $\mathrm{A} 3 \mathrm{C}$ and late diastolic strain rate (SRA) at A3C in the ERP subjects were significantly lower than those in the subjects without ERP. Furthermore, LV basal segment circumferential SRS and SRE were significantly lower in ERP subjects compared to subjects without ERP. Conclusion: Our study suggests that ERP can be more associated with impaired LV longitudinal function than LV circumferential function. In addition, both LV inferolateral region and LV basal segment can be more affected functionally in ERP subjects.
\end{abstract}

\section{Introduction}

Early repolarization pattern (ERP) is defined as an elevation of the $\mathrm{J}$ point in at least 2 leads, as either QRS slurring and notching ${ }^{1}$. For decades, it was believed to be a benign electrocardiographic manifestation ${ }^{2,3}$. Since the early 2000s, this view has been changed based on sporadic observations that linked the J wave with ventricular arrhythmias

1,4,5. The pathophysiological mechanism of arrhythmic events in ERP was primarily explained by electrophysiological alterations ${ }^{6-8}$. Besides, recent studies have demonstrated a significant association between ERP and myocardial structural changes including increased left ventricular (LV) mass and change in LV geometry as well as functional LV alterations such as impaired LV filling ${ }^{9,10}$. Although the relationship between ERP and myocardial structural changes clearly has been shown, it is not exact whether ERP own leads to myocardial dysfunction.

2D speckle tracking echocardiography (2D-STE) has higher diagnostic value in detecting subtle myocardial dysfunction than 2D- transthoracic echocardiography (TTE) ${ }^{11-13}$. This is due to the most important reason is that 2D- STE is more sensitive to make differentiation of passive and active myocardial displacement by tracking small myocardial speckles and following them from frame to frame over cardiac cycle ${ }^{11}$. In ERP individuals, STE can facilitate to detect a silent substrate for arrhythmic events as well as the findings of STE can be used for risk stratification of ventricular arryhtmias in the future. In this study, we aimed to 
investigate LV function by the using 2D-STE in individuals with ERP and to compare these findings with individuals who have normal electrocardiographic pattern.

\section{Method}

\section{Study Population}

A total of 50 healthy individuals with ERP on the surface electrocardiography (ECG) and 50 healthy individuals without ERP who admitted to our cardiology department to get a health report for participating in competitive sports was aged [?]18 years and had no cardiovascular risk factors such as hypertension, hyperlipidemia and diabetes mellitus were included in this study. Written informed consent was obtained from all participants and the study was approved by the local Ethics committee of our institution. The study was conducted in full accordance with the Declaration of Helsinki.

\section{Electrocardiographic Data}

Each participant underwent 12 lead ECG after 10 minutes of resting in the supine position at $25 \mathrm{~mm} / \mathrm{s}$ speed, with a $0.16-100 \mathrm{~Hz}$ filter range and $10 \mathrm{~mm} / \mathrm{mV}$ height. Electrocardiographic examinations were analyzed by two independent cardiologists. ERP was defined according to the definition of Haissaguerre et al. It was evaluated as a $\mathrm{J}$ point elevation which has to be at least $0.1 \mathrm{mV}$ above the isoelectric segment in at least 2 contiguous leads, as well as has the presence of QRS slurring or notching at the QRS terminal ${ }^{1}$. Participants were divided into two groups according to the presence of ERP on the surface ECG: Group 1 was defined as individuals with ERP whereas Group 2 was defined as individuals without ERP. The inter-observer and intra-observer concordance rate for the presence of ERP were $98.5 \%$ and $98.9 \%$, respectively.

\section{Echocardiographic Image Acquisition}

All echocardiographic studies were performed in the left lateral decubitis position with ECG monitoring. Echocardiographic data were obtained from all participants by the same cardiologist using Philips EPIQ-7C Ultrasound System for Cardiology (Andover, USA) with X5-1 probe. Images were digitally stored for offline analysis.

\section{Standart Two Dimensional and Doppler Echocardiographic Data}

LV, left atrial (LA), right ventricular (RV) and aortic root (AoR) dimensions and left ventricular wall thickness (LVWT) were measured according to American Society of Echocardiography 2015 guideline of "Recommendations for Cardiac Chamber Quantification by Echocardiography in Adults" ${ }^{14}$. The relative wall thickness (RWT) was measured as $2 *$ Posterior Wall Thickness/LV-end diastolic diameter. LV mass was calculated from the parasternal view on the basis of Devereux formula ${ }^{15}$.

$\mathrm{E}$ and A wave velocities were measured by pulsed wave Doppler (PW) imaging with the 1- $3 \mathrm{~mm}$. sample volume placed at the mitral leaflet tips in three consecutive cycles. Tissue Doppler imaging (TDI) was used to obtain systolic velocity, early and diastolic relaxation velocities at the septal and lateral mitral annulus from the apical 4 chamber view in three consecutive cycles. Left ventricular ejection fraction (LVEF) was calculated using the modified Simpson's method.

\section{D- Speckle Tracking Strain Data}

STE was conducted according to the recommendations of the American Society of Echocardiography and the European Association of Cardiovascular Imaging ${ }^{16}$. Apical 4-, 3-, 2- chamber views for longitudinal deformation parameters of LV and parasternal short axis (PSAX) views from base to apex for circumferential deformation parameters of LV were obtained and stored for offline analysis in three consecutive cycles. Strain measurement was assessed offline from obtained 2D echocardiographic images using dedicated software with frame rates of [?] 60 frame per second. Firstly, apical 3- chamber (A3C) was selected to determine aortic valve closure time. Endocardial and epicardial border were tracked automatically for A3C, and then automatic tracking of endocardial and epicardial borders were checked carefully. Furthermore, endocardial-epicardial 
tracking and analysis were performed for both apical 4- chamber (A4C) and apical 2- chamber (A2C). 2Dpeak longitudinal systolic strain, peak longitudinal systolic strain rate $\left(\mathrm{SR}_{\mathrm{S}}\right)$, longitudinal early diastolic strain rate $\left(\mathrm{SR}_{\mathrm{E}}\right)$ and longitudinal late diastolic strain rate $\left(\mathrm{SR}_{\mathrm{A}}\right)$ were analyzed for the 17 segments from all of the apical views. The result of analysis was shown as either bullseye map (Figure 1A) and longitudinal strain, strain rate (SR) curves (Figure 1B, 1C). For circumferential strain analysis, apical, mid and basal PSAX views were selected. Endocardial and epicardial border were traced automatically for each segments and then contour tracing was checked and confirmed. The software calculated automatically circumferential systolic strain, circumferential peak $\mathrm{SR}_{\mathrm{S}}$, circumferential $\mathrm{SR}_{\mathrm{E}}$ and circumferential $\mathrm{SR}_{\mathrm{A}}$ for each 16 segments from entire transmural LV of PSAX views. Like longitudinal strain analysis, the results of circumferential strain analysis was presented in a bullseye map or strain /SR curves.

\section{Statistical Analysis}

All analyses were performed with SPSS 25.0 (IBM Corporation, Armonk, NY, USA). Gaussian distribution of continuous variables were confirmed by Shapiro-Wilk test and values were presented as mean with standard deviation. Categorical variables were explained as number or percentage. Comparisons between ERP and control groups were performed using studentt- test for continuous variables, Mann Whitney U test for non-continuous variables, as appropriate. A two- sided $p$ value of $<0.05$ was considered to be statistically significant.

\section{Results}

A total of 50 healthy individuals with ERP and 50 healthy individuals without ERP were included in this study. The frequency of male gender was significantly higher in individuals with ERP compared to individuals without ERP (Table 1).

\section{Standard Two Dimensional and Doppler Echocardiographic Results}

The standard two dimensional echocardiographic characteristics of the study groups are presented in Table 2. RVd, inferolateral wall dimension (ILWd), AoRd, LAd, RWT, LV mass, LV end-diastolic volume (LVEDV) and LV end-systolic volume (LVESV) were significantly higher in individuals with ERP compared to individuals without ERP group. However, E/e' was not significantly different between two groups.

\section{D- Speckle Tracking Longitudinal Strain Results}

The mean value of GLS was $-21.65 \pm 2.95$. Divided into groups with and without ERP, GLS was significantly lower in those with ERP compared to those without ERP $(\mathrm{p}<0.0001)$. The difference in strain persisted across segmental strain values obtained at the $\mathrm{A} 4 \mathrm{C}(\mathrm{p}<0.0001), \mathrm{A} 3 \mathrm{C}(\mathrm{p}<0.0001)$ and $\mathrm{A} 2 \mathrm{C}(\mathrm{p}<0.0001)$ (Figure 2). Also, $\mathrm{SR}_{\mathrm{S}}$ at the $\mathrm{A} 3 \mathrm{C}(\mathrm{p}: 0.011)$ as well as both $\mathrm{SR}_{\mathrm{E}}$ at the $\mathrm{A} 3 \mathrm{C}(\mathrm{p}:<0.0001)$ and $\mathrm{SR}_{\mathrm{A}}$ at the A3C (p:0.034) were significantly lower in individuals with ERP compared to those without ERP. No significant differences were observed in other segments according to $\mathrm{SR}_{\mathrm{S}}, \mathrm{SR}_{\mathrm{E}}$ and $\mathrm{SR}_{\mathrm{A}}$ (Table 3).

\section{D- Speckle Tracking Circumferential Strain Results}

The mean value of GCS was estimated $-23.97 \pm 4.56$. No significant differences were observed with regard to circumferential strain and SR parameters between two groups except LV basal segment $\mathrm{SR}_{\mathrm{S}}$ and $\mathrm{SR}_{\mathrm{E}}$. $\mathrm{LV}$ basal segment circumferential $\mathrm{SR}_{\mathrm{S}}(\mathrm{p}: 0.002)$ and circumferential $\mathrm{SR}_{\mathrm{E}}(\mathrm{p}: 0.006)$ were significantly lower in ERP group (Table 4).

\section{Discussion}

The main findings of our study were as follows: (1) Individuals with ERP had significantly higher LV dimensions, LV wall thickness, RV dimension; (2) a significant inverse interaction was observed between ERP and GLS; (3) ERP had an impact on regional longitudinal and circumferential SR values. The lower longitudinal $\mathrm{SR}_{\mathrm{S}}, \mathrm{SR}_{\mathrm{E}}$ and $\mathrm{SR}_{\mathrm{A}}$ were mainly found at the $\mathrm{A} 3 \mathrm{C}$ and the lower circumferential $\mathrm{SR}_{\mathrm{S}}$ and $\mathrm{SR}_{\mathrm{E}}$ were mainly seen in the basal segment of LV. To our knowledge, this is the first study for demonstrating an important relationship between ERP and both LV longitudinal and circumferential strain and SR parameters. 
ERP is associated with an increased risk of sudden cardiac arrest due to idiopathic ventricular fibrillation in the general population ${ }^{1,5,17}$. Unfortunately, these arrhythmic events can be seen in the young people without cardiac disease such as ischemic heart diseases, cardiomyopathies ${ }^{18}$. However, Trenkwalder et al. and McNamara et al. showed that the morphological changes of LV began to be seen in ERP subjects ${ }^{9,10}$. In these subjects, ventricular dysfunction can not be determined with the use of conventional echocardiographic modalities ${ }^{18,19}$. STE and TDI can clearly detect subtle ventricular dysfunction by calculating the ventricular strain and SR which are more sensitive indicators than LVEF ${ }^{20,21}$.

In our study, we found that both LV and RV dimensions, and LV wall thickness were significantly increased in subjects with ERP. In addition, it was observed that LV mass and RWT were significantly higher in ERP group. Our results are consistent with the previous studies ${ }^{9,22,23}$. These results suggest that ERP can lead to LV structural changes. As we known, LV radius, LV wall thickness and hemodynamic conditions impose onto LV- wall stress (WS ${ }^{24}$. In ERP group, increased LV volumes may lead to proliferation of LV myocardium to normalize LV-WS. These structural changes may be accompanied by oxygen demand- supply mismatch which leads to myocardial systolic dysfunction, even before LVEF reduces.

The most important finding of our study was that ERP participants had a significantly lower GLS. There are plausible mechanisms for why ERP produced impaired GLS. Firstly, ERP can mediate impaired GLS through its effects on LV structural changes. Especially, the increased LVESV and LVEDV can lead to increased WS followed by impaired GLS. Because, ventricular strain and SR has been known as WS dependent indicators. In line with our work, several studies have also reported a significant inverse relationship between WS and strain and SR parameters ${ }^{25-28}$. The second mechanism for impaired GLS values may be that mutations in genes can cause both ion channel dysfunction and myocardial dysfunction ${ }^{29,30}$. Chen et al. showed that CACNA1C/ DES/ MYPN mutations are the pathogenic substrates for the clinical manifestation in the hypertrophic cardiomyopathy patients with ERP ${ }^{29}$. ERP could be a heritable condition with variable penetrance and incomplete phenotypic manifestation. In such patients, impaired GLS may also guide further genetic analysis. To our knowledge, our study was the first to evaluate the relationship between GLS and ERP and also to explain the possible underlying mechanisms for impaired GLS in ERP subjects. However, unlike to these findings, Gulel et al. reported that myocardial deformation parameters including longitudinal and circumferential S/ SR were not affected by ERP ${ }^{31}$. The main reason for this unexpected result in Gulel et al. may be due to lower number participants in their study. In addition, mean age was lower in Gulel's study. The possibility of ERP induced morphological and functional changes on the myocardium may increased with advancing age.

The significantly lower longitudinal $\mathrm{SR}_{\mathrm{S}} \mathrm{SR}_{\mathrm{E}}$ and $\mathrm{SR}_{\mathrm{A}}$ at the $\mathrm{A} 3 \mathrm{C}$ in $\mathrm{ERP}$ can be consistent with the significantly higher IL wall dimension in ERP group due to WS relationship. In addition, these findings may be consistent with the animal studies which showed higher levels of transient outward potassium current $\left(\mathrm{I}_{\mathrm{to}}\right)$ in the inferior wall and lateral wall of canine myocardium ${ }^{32}$. Because, long non- coding RNAs which has been known as myocardial $\mathrm{K}+$ channel regulators may play an important role in subtle myocardial dysfunction in the region with higher $\mathrm{I}_{\text {to }}$ density ${ }^{33}$.

The association between ERP and impaired basal segment circumferential $\mathrm{SR}_{\mathrm{S}}$ and $\mathrm{SR}_{\mathrm{E}}$ deserves further comment. We previously explained that the morphological changes related to ERP could be affected by ion channel density. The density of potassium $(\mathrm{K}+)$ channel varies from apical segment to basal segment of $\mathrm{LV}^{34,35}$. The density of $\mathrm{K}+$ channel in apical segment is approximately twice as high as in basal segment ${ }^{35}$. In accordance with our previous findings, in ERP group, the lower frequency of basal segment ion channel density may be associated with a thinner wall thickness in basal segment than that in apical segment. In addition, basal segment has the largest radius as compared to mid and apical segment of LV. The combined effect of basal segment's ion channel density and large radius may cause significantly higher WS in the basal segment of LV in subjects with ERP than those without ERP, resulting in the fact that LV basal segment needs more energy for both contraction and relaxation than mid and apical segment do. Consequently, the presence of ERP accompanied by worse basal segment circumferential $\mathrm{SR}_{\mathrm{S}}$ and $\mathrm{SR}_{\mathrm{E}}$.

\section{Limitations}


There were several limitations in our study. First, this was a single center study and the sample size was relatively small. Second, our study was a short term study. It could be interesting to follow up the individuals with ERP in terms of arrhythmic events. Finally, we did not evaluate rotational parameters such as twist and torsion.

\section{Conclusion}

In this study, we found that ERP can affect subtle myocardial dysfunction especially in IL wall and LV basal segment. This relationship can be consistent with the $\mathrm{K}+$ channel density and $\mathrm{K}+$ channel distribution in LV myocardium. In addition, ERP can be associated with more impaired longitudinal function than circumferential function. We therefore propose that ERP not only can lead to ion channel dysfunction but also result in myocardial dysfunction.

\section{References}

1. Haissaguerre M, Derval N, Sacher F, et al. Sudden cardiac arrest associated with early repolarization. The New England journal of medicine. 2008;358(19):2016-2023.

2. Macfarlane PW, Antzelevitch C, Haissaguerre M, et al. The Early Repolarization Pattern: A Consensus Paper. Journal of the American College of Cardiology. 2015;66(4):470-477.

3. Bourier F, Denis A, Cheniti G, et al. Early Repolarization Syndrome: Diagnostic and Therapeutic Approach. Frontiers in cardiovascular medicine. 2018;5:169.

4. Tikkanen JT, Anttonen O, Junttila MJ, et al. Long-term outcome associated with early repolarization on electrocardiography. The New England journal of medicine. 2009;361(26):2529-2537.

5. Rosso R, Kogan E, Belhassen B, et al. J-point elevation in survivors of primary ventricular fibrillation and matched control subjects: incidence and clinical significance. Journal of the American College of Cardiology. 2008;52(15):1231-1238.

6. Yan GX, Antzelevitch C. Cellular basis for the electrocardiographic J wave. Circulation. 1996;93(2):372379 .

7. Abe A, Ikeda T, Tsukada T, et al. Circadian variation of late potentials in idiopathic ventricular fibrillation associated with $\mathrm{J}$ waves: insights into alternative pathophysiology and risk stratification. Heart rhythm. 2010;7(5):675-682.

8. Gussak I, Antzelevitch C. Early repolarization syndrome: clinical characteristics and possible cellular and ionic mechanisms. Journal of electrocardiology. 2000;33(4):299-309.

9. McNamara DA, Bennett AJ, Ayers C, de Lemos JA, Berry JD, Link MS. Early Repolarization Pattern Is Associated With Increased Left Ventricular Mass: Insights From the Dallas Heart Study. JACC Clinical electrophysiology. 2019;5(3):395-397.

10. Trenkwalder T, Rübsamen N, Schmitt VH, et al. Left ventricular geometry and function in early repolarization: results from the population-based Gutenberg Health Study. Clinical Research in Cardiology. 2019 .

11. Collier P, Phelan D, Klein A. A Test in Context: Myocardial Strain Measured by Speckle-Tracking Echocardiography. Journal of the American College of Cardiology. 2017;69(8):1043-1056.

12. Sun M, Dong Y, Wang Y, Li G, Huang D. Assessment of the left ventricular function in patients with uremia using layer-specific 2-dimensional speckle tracking echocardiography. Medicine.2019;98(9):e14656.

13. A Reisner S, Lysyansky P, Agmon Y, Mutlak D, Lessick J, Friedman Z.Global longitudinal strain: A novel index of left ventricular systolic function. Vol 172004.

14. Lang RM, Badano LP, Mor-Avi V, et al. Recommendations for cardiac chamber quantification by echocardiography in adults: an update from the American Society of Echocardiography and the European Asso- 
ciation of Cardiovascular Imaging. Journal of the American Society of Echocardiography : official publication of the American Society of Echocardiography. 2015;28(1):1-39.e14.

15. Devereux RB, Alonso DR, Lutas EM, et al. Echocardiographic assessment of left ventricular hypertrophy: comparison to necropsy findings. The American journal of cardiology. 1986;57(6):450-458.

16. Mor-Avi V, Lang RM, Badano LP, et al. Current and evolving echocardiographic techniques for the quantitative evaluation of cardiac mechanics: ASE/EAE consensus statement on methodology and indications endorsed by the Japanese Society of Echocardiography. Journal of the American Society of Echocardiography : official publication of the American Society of Echocardiography. 2011;24(3):277-313.

17. Wu SH, Lin XX, Cheng YJ, Qiang CC, Zhang J. Early repolarization pattern and risk for arrhythmia death: a meta-analysis. Journal of the American College of Cardiology. 2013;61(6):645-650.

18. Takagi M, Aihara N, Takaki H, et al. Clinical characteristics of patients with spontaneous or inducible ventricular fibrillation without apparent heart disease presenting with $\mathrm{J}$ wave and ST segment elevation in inferior leads. Journal of cardiovascular electrophysiology.2000;11(8):844-848.

19. Aagaard P, Braunschweig F, Wecke L, Sahlen A, Bergfeldt L. Early repolarization in middle-age runners: cardiovascular characteristics. Medicine and science in sports and exercise.2014;46(7):1285-1292.

20. Potter E, Marwick TH. Assessment of Left Ventricular Function by Echocardiography: The Case for Routinely Adding Global Longitudinal Strain to Ejection Fraction. JACC Cardiovascular imaging.2018;11(2 Pt 1):260-274.

21. Smiseth OA, Torp H, Opdahl A, Haugaa KH, Urheim S. Myocardial strain imaging: how useful is it in clinical decision making? European heart journal. 2016;37(15):1196-1207.

22. Miragoli M, Goldoni M, Demola P, et al. Left ventricular geometry correlates with early repolarization pattern in adolescent athletes.Scandinavian journal of medicine 83 science in sports. 2019.

23. Reinhard W, Trenkwalder T, Haller B, et al. The early repolarization pattern: Echocardiographic characteristics in elite athletes. Annals of noninvasive electrocardiology : the official journal of the International Society for Holter and Noninvasive Electrocardiology, Inc. 2019;24(2):e12617.

24. Badeer HS. CONTRACTILE TENSION IN THE MYOCARDIUM. American heart journal. 1963;66:432434.

25. Aurigemma GP, Silver KH, Priest MA, Gaasch WH. Geometric changes allow normal ejection fraction despite depressed myocardial shortening in hypertensive left ventricular hypertrophy. Journal of the American College of Cardiology. 1995;26(1):195-202.

26. Shimizu G, Hirota Y, Kita Y, Kawamura K, Saito T, Gaasch WH. Left ventricular midwall mechanics in systemic arterial hypertension. Myocardial function is depressed in pressure-overload hypertrophy. Circulation. 1991;83(5):1676-1684.

27. DeVore AD, McNulty S, Alenezi F, et al. Impaired left ventricular global longitudinal strain in patients with heart failure with preserved ejection fraction: insights from the RELAX trial. European journal of heart failure. 2017;19(7):893-900.

28. Pellicori P, Kallvikbacka-Bennett A, Khaleva O, et al. Global longitudinal strain in patients with suspected heart failure and a normal ejection fraction: does it improve diagnosis and risk stratification? The international journal of cardiovascular imaging. 2014;30(1):69-79.

29. Chen Y, Barajas-Martinez H, Zhu D, et al. Novel trigenic CACNA1C/DES/MYPN mutations in a family of hypertrophic cardiomyopathy with early repolarization and short QT syndrome. Journal of translational medicine. 2017;15(1):78. 
30. Ackerman MJ, Priori SG, Willems S, et al. HRS/EHRA expert consensus statement on the state of genetic testing for the channelopathies and cardiomyopathies: this document was developed as a partnership between the Heart Rhythm Society (HRS) and the European Heart Rhythm Association (EHRA). Europace : European pacing, arrhythmias, and cardiac electrophysiology : journal of the working groups on cardiac pacing, arrhythmias, and cardiac cellular electrophysiology of the European Society of Cardiology. 2011;13(8):1077-1109.

31. Gulel O, Dagasan G, Yuksel S, Soylu K, Sahin M. Evaluation of left ventricular myocardial deformation parameters in individuals with electrocardiographic early repolarization pattern. Anatolian journal of cardiology. 2016;16(11):850-854.

32. Mercer BN, Begg GA, Page SP, Bennett CP, Tayebjee MH, Mahida S. Early Repolarization Syndrome; Mechanistic Theories and Clinical Correlates. Frontiers in physiology. 2016;7:266.

33. Yang KC, Nerbonne JM. Mechanisms contributing to myocardial potassium channel diversity, regulation and remodeling. Trends in cardiovascular medicine. 2016;26(3):209-218.

34. Opthof T, Remme CA, Jorge E, et al. Cardiac activation-repolarization patterns and ion channel expression mapping in intact isolated normal human hearts. Heart rhythm.2017;14(2):265-272.

35. Szentadrassy N, Banyasz T, Biro T, et al. Apico-basal inhomogeneity in distribution of ion channels in canine and human ventricular myocardium. Cardiovascular research. 2005;65(4):851-860.

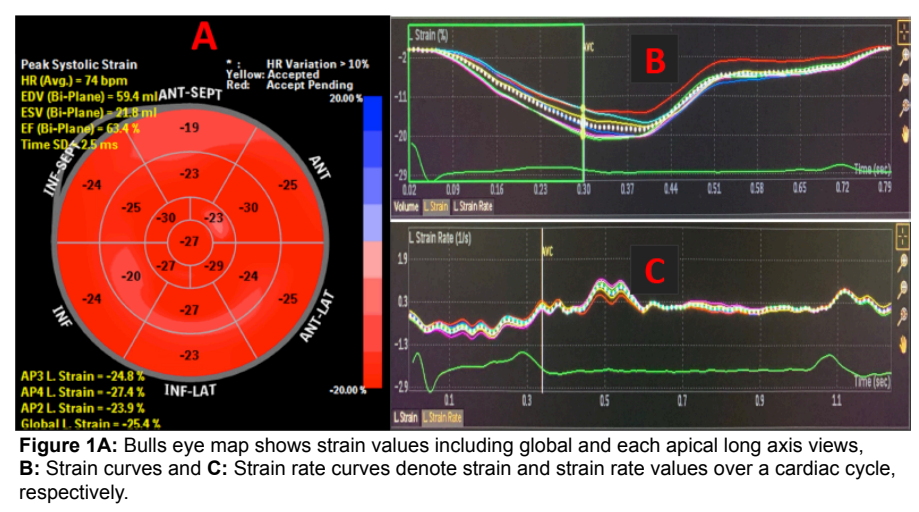




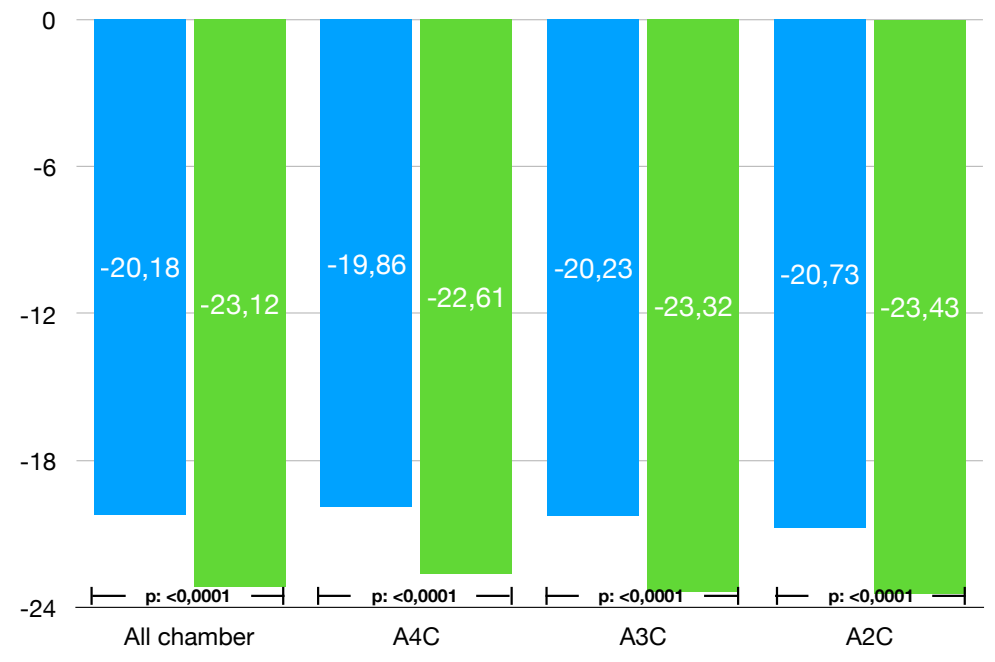

Figure 2: The comparison of global longitudinal strain according to presence of early repolarization pattern. Blue line: early repolarization pattern (+); green line: early repolarization pattern (-). 